\title{
Data mining algorithms in the task of diagnosing the welded joints quality
}

\author{
R R Akhmedyanov ${ }^{1}$, K F Tagirova ${ }^{1}$, A M Vulfin ${ }^{1}$, V V Berkholts ${ }^{1}$ \\ and R Ch Gayanov ${ }^{2}$ \\ ${ }^{1}$ Ufa State Aviation Technical University, K. Marks st., 12, Ufa, Russia, 450077 \\ ${ }^{2}$ Higher School of Economics, Myasnitskaya str., 20, Moscow, Russia, 101000 \\ e-mail: vulfin.alexey@gmail.com
}

\begin{abstract}
The paper discusses the issue of creating an intelligent diagnostic system for welded joints based on the radiographic method. This will speed up the process of decoding radiographic images and reduce the number of errors associated with human factors, since at this time most of the work on decoding images is done manually.

The goal of the work is to develop an intelligent system for finding defects in a welded joint in a radiographic image using neural networks. The obtained results are the algorithm of operation of the intelligent diagnostic system for welded joints based on the radiographic method, a trained neural network for detecting defects of welded joints.
\end{abstract}

\section{Introduction}

When performing welding work, it is obligatory according to the current regulatory documents to conduct radiographic inspection of welded joints. The most common type of radiographic inspection is to obtain X-rays of the welds. The conclusion about the quality of the test compound is made by a flaw detector technologist based on the results of X-ray image decoding. Most of the mistakes made during such control are due to the fatigue of people employed in repeated visual checks.

The object of the study are welded joints made by melting the metals being welded. The subject of research is the quality control of welded joints using an intelligent system.

The goal of the work is the development, research and implementation of digital image processing algorithms in the system for diagnostics of welded joints based on the radiographic method.

To achieve the goal, the following tasks were set:

- Development of the structure of the defects recognition system in welded joints based on the analysis of images of radiographic images of welded joints using a neural network;

- Development of an algorithmic and software implementation of the localization of defects in welded joints using a neural network. 


\section{Development of the structure of the system for detecting defects of welded joints based on image analysis}

Radiographic control (Radio-graphic method of NDT) is a method of radiation non-destructive testing (NTD), based on the transformation of the image of the monitored object into a radiographic image or recording of this image on a memory device and subsequent conversion to a light image $[1,18]$.

Radiographic control is carried out in order to identify surfacing and welded joints (seam and heataffected zone):

- cracks;

- lack of fusion;

- pores;

- metal and non-metallic inclusions, the density of which differs from the density of the welded joint metal (tungsten, slag, oxide, etc.);

- inaccessible for external inspection of undercuts, burn-throughs, etc. [2].

The process of radiographic inspection of a welded joint is shown in Figure 1.

- analysis of signals characterized by a high degree of uncertainty, e.g., "non-stochastic" type, which includes most biomedical signals, including ECS;

- increasing the level of intelligent assistance of medical specialists;

- revealing hidden regularities and extracting new knowledge from the accumulated data, which will allow to build production systems of explaining the diagnostic solutions.

The process of radiographic inspection of a welded joint is shown in Figure 1.

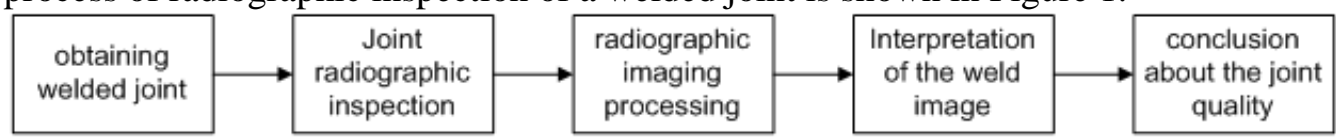

Figure 1. Block diagram of the process of radiographic control.

Interpretation of X-rays of welded joints is carried out in a visual way. This leads to the fact that subjective factors affect the results of the monitoring and, as a result, the quality of the decoding of images and the reliability of the results of the monitoring are significantly reduced. A large amount of work performed by a person leads to the fact that the process of flaw detection takes a long time and lags far behind the performance of the main process - welding of the product.

According to the standard "GOST 30242-97 Defects of compounds during fusion welding. Classification, designations and definitions" [3] defects are classified into six groups. In this research, such defects as "pores", "inclusions" and "lack of fusion" are considered.

Interpretation a radiographic image can be extremely difficult if it is too light or dark. In such a picture it is impossible to detect fine details, which may affect the detectability of the defect. To increase the probability of detecting a defect in a welded joint, it is necessary that the dark and light areas of the image of the welded joint do not exceed the working density range. According to the standards "GOST 7512-82" [4] and the unified methodology for radiographic control "PNAE G-7017-89" optical density of the image should not be less than 1.5 B and not more than $3.5 \mathrm{~B}$.

In case of deviation from specifications and regulatory documents, violations of the welding process, as well as lack of qualifications of the welder, defects in welded joints occur.

According to the standard "GOST 30242-97 Defects of compounds during fusion welding. Classification, designations and definitions" defects in welding metals by fusion are formed due to violation of the requirements of regulatory documents for welding materials, preparation, assembly and welding of the elements being joined, thermal and mechanical processing of welded joints and the design as a whole [11].

In this standard, defects are classified into six groups, as shown in Figure 2.

Cracks are the most dangerous defects of a welded joint; their appearance is not permissible (Figure 3). Cracks are divided into external and internal. 


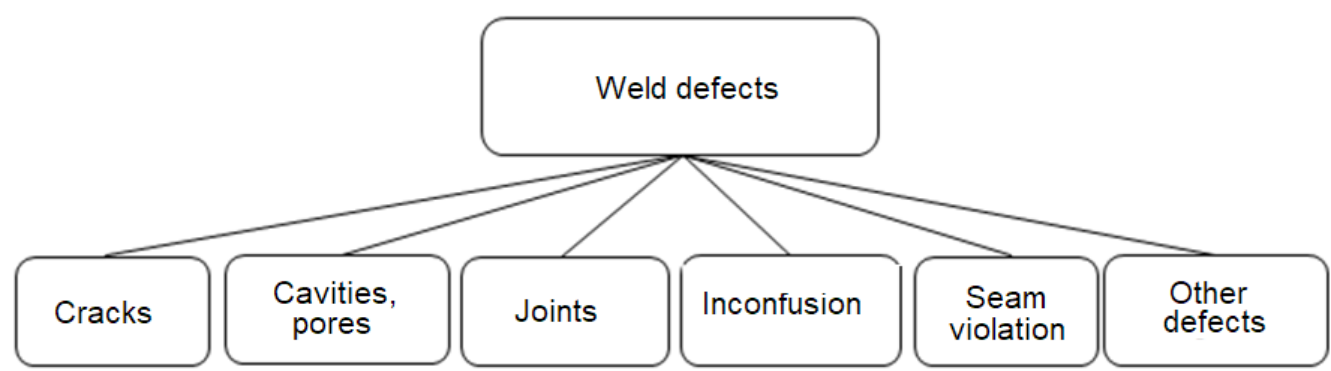

Figure 2. Classification of defects in welded joints.

The formation of cracks occurs as a result of uneven heating, cooling and shrinkage in the metal when welding a welded joint. Also in high carbon and alloy steels, cracks may form as a result of the hardening of the metal after welding [8]. Cracks are divided by size into macro and microscopic ones, and, depending on the origin, into cold and hot. By location: transverse and longitudinal.

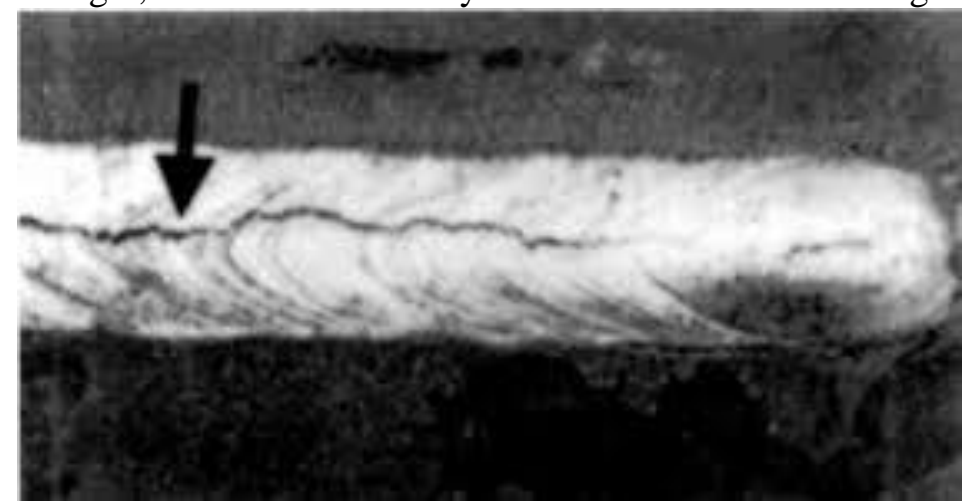

Figure 3. Crack in welded joint.

The formation of internal and surface pores is associated with the ingress of gases, nitrogen, hydrogen, carbon dioxide, etc.) into the weld metal during welding (Figure 4). Pores with nitrogen are formed due to the ingress of atmospheric air into the weld metal with poor-quality protection. Hydrogen is formed due to the ingress of moisture, oils and electrode coating components. Carbon dioxide is formed in the process of welding a welded joint when carbon burns in the metal of the product being welded. The main reason for the appearance of pores is the poor preparation of welded edges, the presence of dirt, rust, moisture, oils, the use of raw electrodes [8].

Pores can be distributed in a welded joint as follows:

- uniformly over the entire length of the welded joint;

- congestion in a separate area;

- chained.

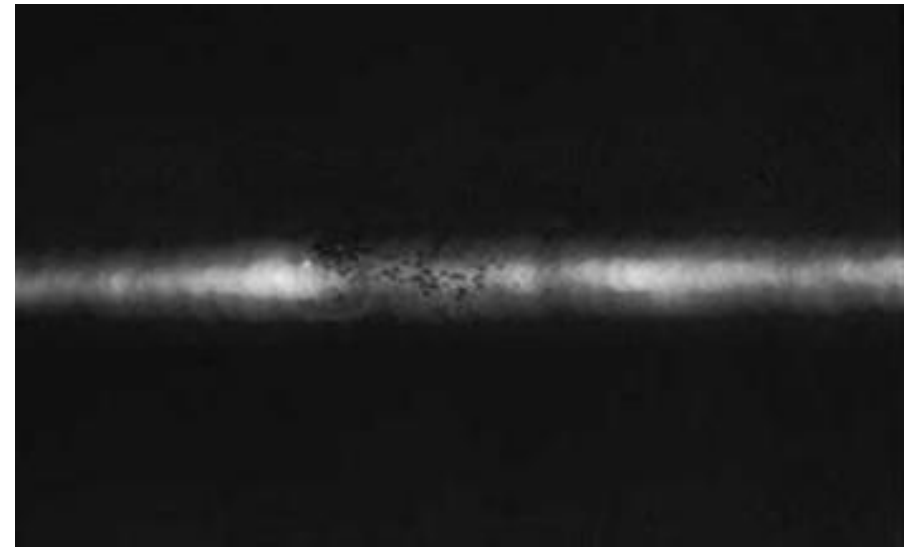

Figure 4. Gas pores accumulation. 
The formation of nonmetallic inclusions is characterized by the use of small welding currents, low quality electrodes, welding wire, flux, as well as when the edges are contaminated and the slag is poorly cleaned from welding during multi-layer welding (Figure 5) [5]. There are the following types of inclusions:

- slag inclusions;

- flux inclusions;

- oxide inclusions;

- metal inclusions.

Negative factors of inclusions are the weakening of the cross section of the seam and the decrease in strength and ductility of the welded joint.

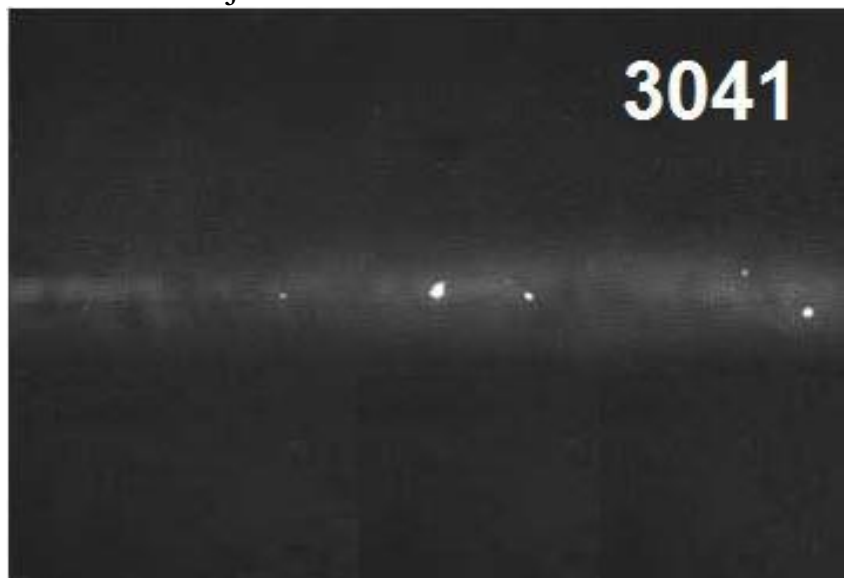

Figure 5. Slag inclusions.

Leakage is a defect in the form of non-fusion in a welded joint (Figure 6) due to incomplete melting of the edges of the base metal or surfaces of previously made weld beads [1].

The sagging is the result of the deposition of the deposited metal on the unheated surface of the base metal or the roller previously made without fusing with it. Such defects can be with low qualification of the welder, poor-quality electrodes and mismatch of the welding speed and welding current to weld seam [8].

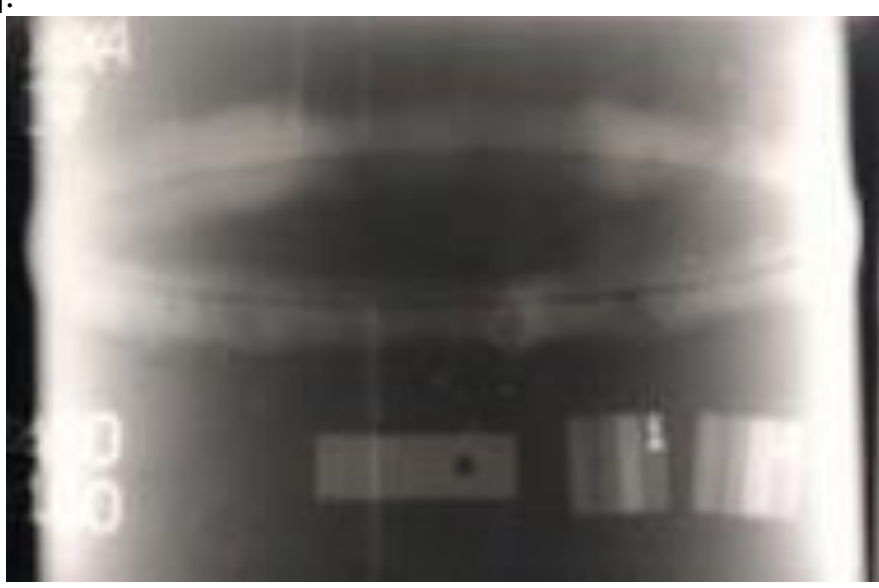

Figure 6. Inconfusion of welted joint.

Violation of the seam shape is expressed in the discrepancy between the height and width of the seam and abrupt transition from the base metal to the weld metal (Figure 7). These defects are the result of poor preparation of welded edges, poor build quality for welding. 


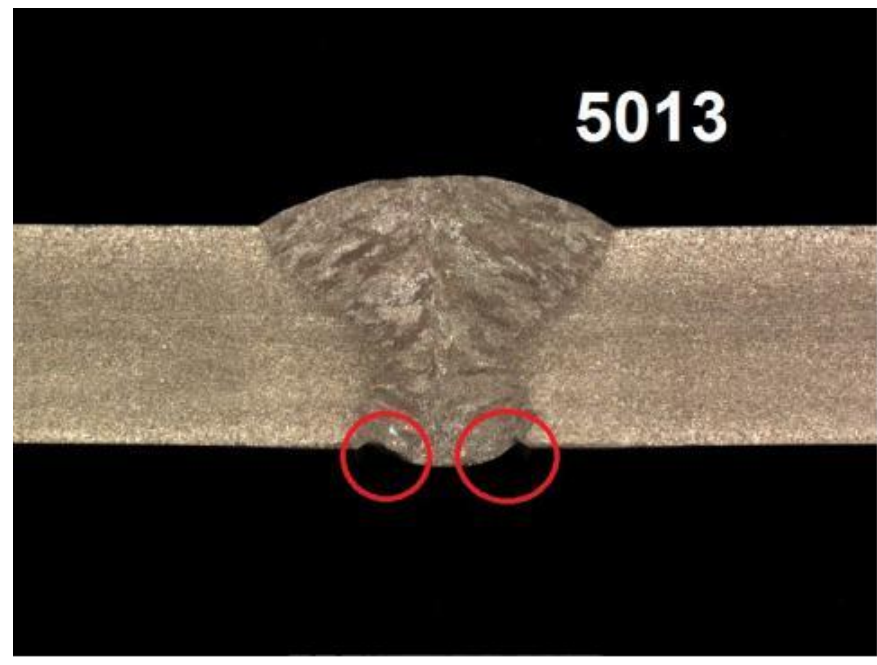

Figure 7. Shrinkage groove.

Violations of the seam shape include the following defects:

- undercuts;

- shrinkage grooves;

- Excess convexity butt and fillet welds;

- excess of the fusion;

- wrong seam profile

- linear and angular displacements of welded elements;

- leaking;

- burnout;

- not completely filled cutting edges;

- excessive asymmetry of the corner seam;

- uneven joint width;

- uneven surface;

- concavity of the root of the weld.

\section{Radiographic image quality analysis}

The quality of the radiographic image is determined by the following parameters:

- optical density of blackening;

- contrast;

- image sharpness.

Decoding a radiographic image can be extremely difficult if it is too light or dark. In such a picture it is impossible to detect fine details, which may affect the detectability of the defect. To improve the quality of detection of a defect in a welded joint, it is necessary that the dark and light areas of the image of the welded joint should not exceed the working density range. According to the standards, GOST 7512-82 and the unified methodology for radiographic monitoring of the PNAE G-7-017-89, the optical density of the image should not be less than $1.5 \mathrm{~B}$ and not more than $3.5 \mathrm{~B}$.

The actual sensitivity of the control depends on the degree of detection of small defects. The defining parameters of the real sensitivity control are the contrast and sharpness of the image.

The contrast of the image in the picture is determined by the formula 1 :

$$
\Delta D_{o}=D_{o 2}-D_{o 1}
$$

where $D_{o 2}$ and $D_{o 1}$ are the optical density of the image parts.

The visual assessment of the contrast of the image depends largely on the human factor, and specifically on the physiological and psychological characteristics. The contrast of the image or the minimum difference between the two areas in the image that the human eye is able to distinguish is $0.02-0.04$. 
The sharpness of the image is determined by sharp transitions between the blackening density of the image areas. The greatest sharpness is characterized by a narrow transition from light to dark areas of the image, which gives a significant distinctiveness of the contours of the object. An image with high sharpness has clear contours of the object of translucence and the desired defects of the welded joint, which ensures their detectability.

With a wide transition between the areas, a blur of the contours of the object is formed, which greatly affects the detectability.

For registration of closely spaced defects, the resolution of the radiographic film is not unimportant. The fine-grained films have the highest resolution, their use ensures a good detectability of defects.

The following factors influence the sensitivity of radiographic monitoring (Table 1):

- geometric conditions of scanning;

- the shape of the defect and its location relative to the direction of transmission;

- X-ray stiffness, thickness and density of the translucent material;

- characteristics of the film and the correctness of its photo processing after exposure;

- The use of reinforcing screens.

The amount of unsharpness is determined by:

- geometric blur;

- internal detector blurriness.

According to PNAE G-7-010-89, images allowed for decoding should meet the following requirements:

- on the image of the seam and the controlled heat-affected zone there should be no spots, stripes, dirt and damage to the emulsion layer;

- the pictures should be visible clear images of restrictive labels, marks and standards of sensitivity (except for cases provided by this method, when the control is carried out without the installation of restrictive marks or marks, or standards of sensitivity, or both);

- the optical density of the images of the controlled areas of the weld and the heataffected zone, as well as the sensitivity standards, should not be less than 1.5 and more than 3.5 (when monitoring welded joints with a variable cross-section, it is possible to increase the optical density of images of the welded joint sections with a minimum thickness of 4.0 );

- reduction of the optical density of the image of the seam and the controlled heataffected zone on any part of this image with respect to the optical density of the image of the sensitivity standard (or the area on which the sensitivity standard is installed) must not exceed 1.0;

- control sensitivity determined from the image of the sensitivity standard (minimum depth of the groove of the groove pattern or minimum wire diameter of the wire standard visible in the picture) must meet the requirements of ПНАЭ Г-7-010-89 [2].

Table 1. Factors affecting the quality of the radiographic image.

\begin{tabular}{|c|c|c|c|}
\hline \multicolumn{2}{|c|}{ Radiographic contrast } & \multicolumn{2}{|c|}{ Resolution } \\
\hline $\begin{array}{l}\text { Contrast due to } \\
\text { transmission }\end{array}$ & Film contrast & Geometric blur & Film grain \\
\hline $\begin{array}{l}\text { Affect: } \\
\text { - product parameters } \\
\text { (density, chemical } \\
\text { composition, } \\
\text { thickness); } \\
\text { - radiation rigidity; } \\
\text { - } \quad \text { - diffuse }\end{array}$ & $\begin{array}{l}\text { Affect: } \\
\text { - type of film; } \\
\text { - technology of photo } \\
\text { processing; } \\
\text { - optical density, the } \\
\text { presence and type of } \\
\text { screens (fluorescent, }\end{array}$ & $\begin{array}{l}\text { Affect: } \\
\text { - the size of the focal } \\
\text { spot; } \\
\text { - distance source - } \\
\text { film: } \\
\text { - distance product - } \\
\text { film, }\end{array}$ & $\begin{array}{l}\text { Affect: } \\
\text { - type of film; } \\
\text { - type of screens; } \\
\text { - radiation rigidity; } \\
\text { - photo processing } \\
\text { technology. }\end{array}$ \\
\hline
\end{tabular}




\begin{tabular}{|l|l|l|}
\hline radiation & $\begin{array}{l}\text { lead); differential } \\
-\quad \text { charging circuit } \\
\text { cassette. }\end{array}$ & $\begin{array}{l}- \\
\text { thickness of the } \\
\text { product; } \\
- \text { the quality of the } \\
\text { contact between the } \\
\text { screens and the film, } \\
- \text { clean screens; } \\
-\quad-\quad \text { object } \\
\text { displacement relative } \\
\text { to film }\end{array}$ \\
\hline
\end{tabular}

Weld seams, images that are not approved for decoding, must be re-examined.

\section{Automation of the process of diagnosing the quality of a welded joint based on the radiographic method}

\subsection{Description of the algorithm of the process of diagnosing the quality of a welded joint based on} radiographic monitoring

The block diagram of the system of diagnostics of welded joints is shown in Figure 3, the algorithm of the system is shown in Figure 8.

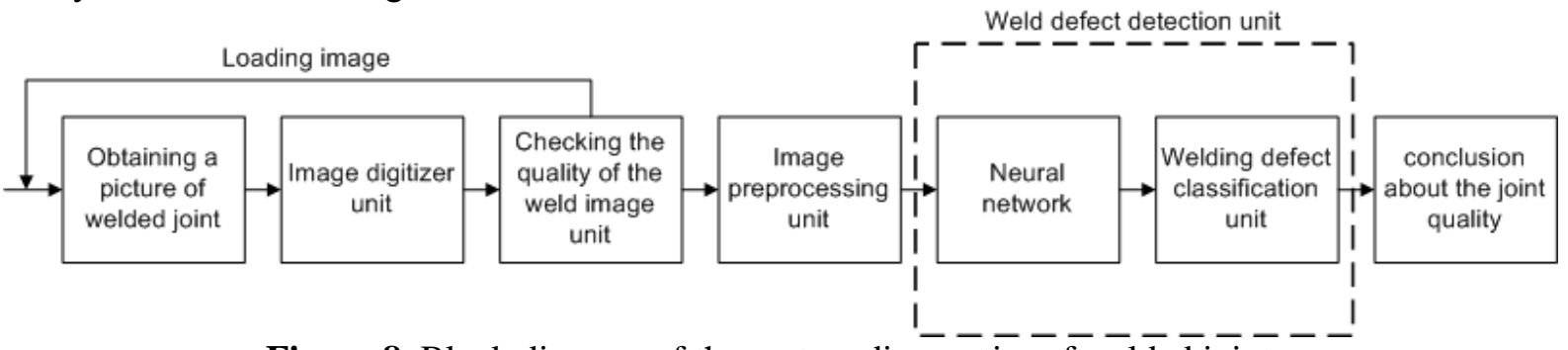

Figure 8. Block diagram of the system diagnostics of welded joints.

The image obtained during digitization must be transferred from color to halftone, noise must be removed and the contrast of the image must be increased for further work.

Images entered into a computer are often low-contrast. Often, low contrast is caused by a large range of reproducible brightness with non-linearity of the level transfer characteristic. The dependence of the brightness of pixels on the minimum value to the maximum also affects the image quality. The optimal is the linear form of the function of changing the intensity of the brightness of pixels. With a convex characteristic, the image will be brighter, and with a more concave dark. In both cases, the attributes of objects can be distorted and their recognition can be difficult. Correcting the brightness of the image significantly improves its quality.

\subsection{Algorithm of the process of determining the weld image quality}

In radiographic control, one of the stages preceding the decoding of the image is the determination of the quality of the image and its compliance with all the necessary requirements. One of the requirements is the compliance of the optical density of the images of the controlled areas of the weld and the heat-affected zone, as well as the standards of sensitivity (Figure 10).

The ratio of the amount of incoming light to the amount of light passing through a certain area is called the optical density of the film and is determined by the formula (2):

$$
D=\log \frac{I_{0}}{I_{1}}
$$

where $D$ - optical density; $I_{0}$ - intensity of light falling on the film; $I_{1}$ - intensity of light emanating from this part of the film. 


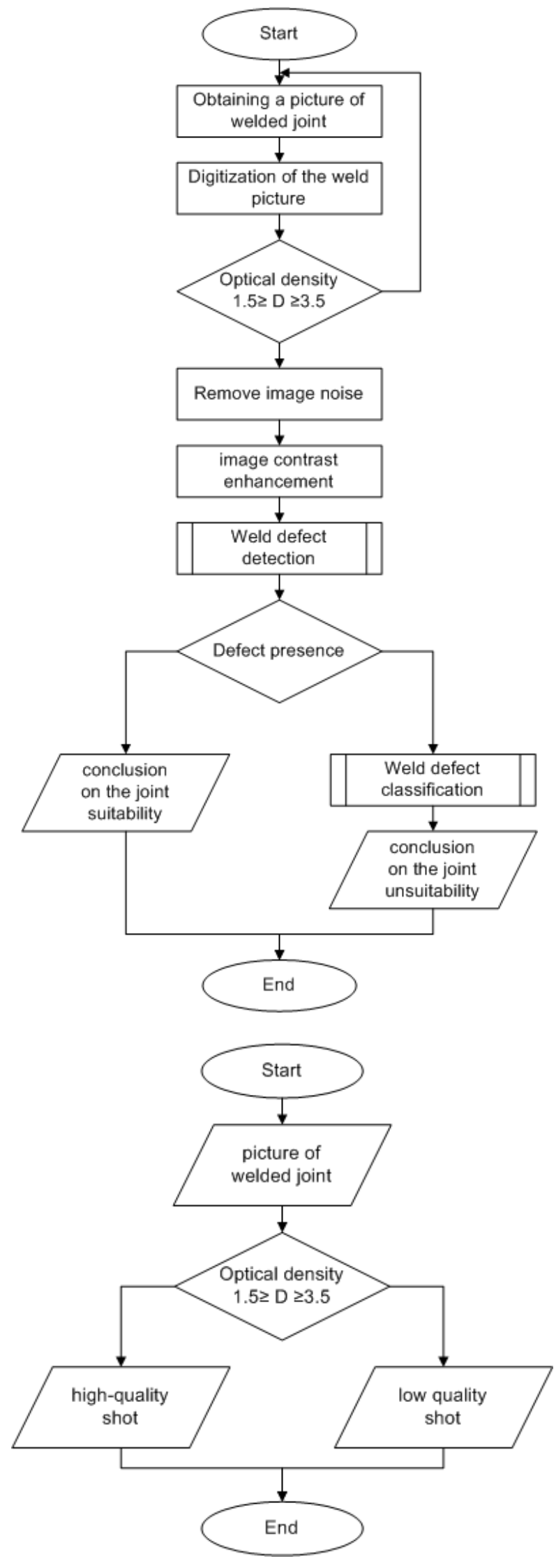

Figure 9. Graphic diagram of the algorithm for diagnosing a welded joint based on the radiographic method.

Figure 10. Algorithm for assessing the quality of a radiographic image. 
The dependence of optical density and exposure is represented as a graphical characteristic. The curve reflects the ratio of the logarithms of the relative exposures to the resulting optical densities of the blackening of the film (Figure 11).

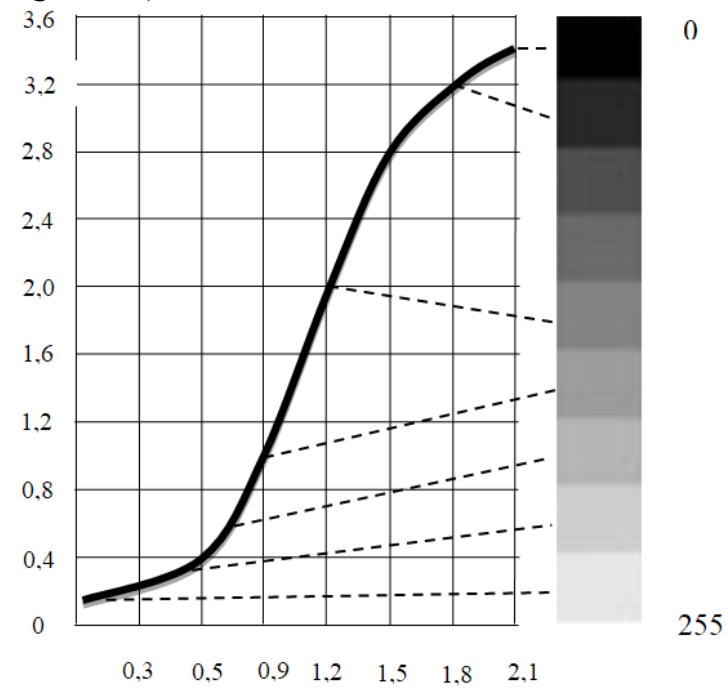

Figure 11. The characteristic curve of the $x$-ray film.

To automate the process of identifying compliance with the requirements of X-ray films, digitization of the image is necessary. After digitization, it is necessary to convert color (RGB) images to halftone. In a grayscale image, pixel brightness has a uniform scale from 0 to 255 . Accordingly, optical density can be represented in arbitrary brightness units, where the value 0 corresponds to the darkest part of the image (black) and 255 the brightest part of the image (white).

According to "PNAE G-7-010-89" [5], the optical density of images of monitored sections of the weld and heat affected zone, as well as sensitivity standards, should not be less than 1.5 and more than 3.5. Accordingly, it is possible to distinguish the working range of optical densities and the corresponding pixel brightness in the zones of monitored areas (Figure 12).

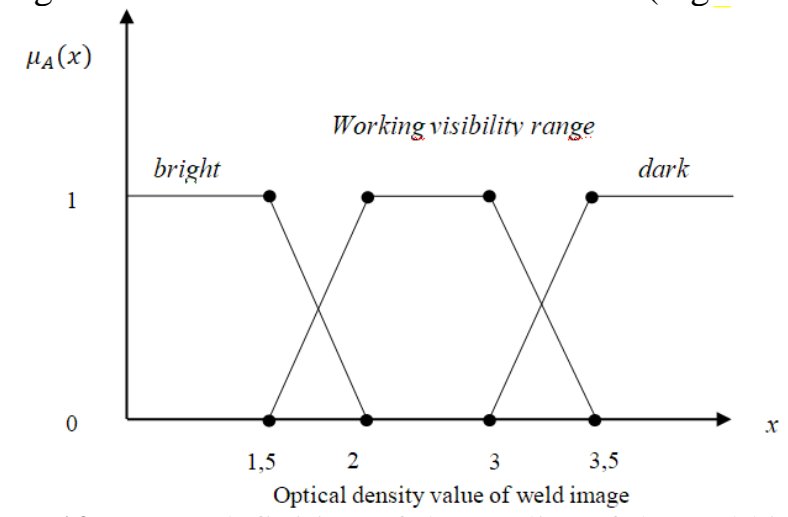

Figure 12. Fuzzy definition of the quality of the weld image.

The graph shows that the useful density range is in the range from 2 to 3 Bell, which corresponds to a pixel brightness of 40 units to 130 . The working brightness range provides maximum visibility of defects in welded joints, which significantly affects the control results. Images whose control areas are beyond the allowable values according to "PNAE G-7-010-89" less than 1.5 and more than 3.5 Bell, which is in the pixel brightness range above 155 units and below 20, are considered non-compliant. Control areas of welds whose images did not pass quality control are subject to repeated radiographic control. 


\section{Experimental studies of the automated system for diagnosing the quality of welded joints}

To test the developed recognition algorithms, a compiled database of images of radiographic images of welded joints was used, containing images of defects, as well as corresponding tags. For the training of classifiers 900 images were used, each image in the area of the defect is marked with labels of the area of interest (ROI label).

Three types of markers of areas of interest (ROI) were created corresponding to defects on the image array of welded joints:

- lack of fusion;

- pores;

- inclusions;

Marks of interest were placed on each image with the intended location of the corresponding defect (Figure 13-15).

A defect in the form of a "pore" is characterized by a small area of darkening of a spherical shape on the image of a welded joint, in most cases in this sample of images of welded joints, the pores are in a group.

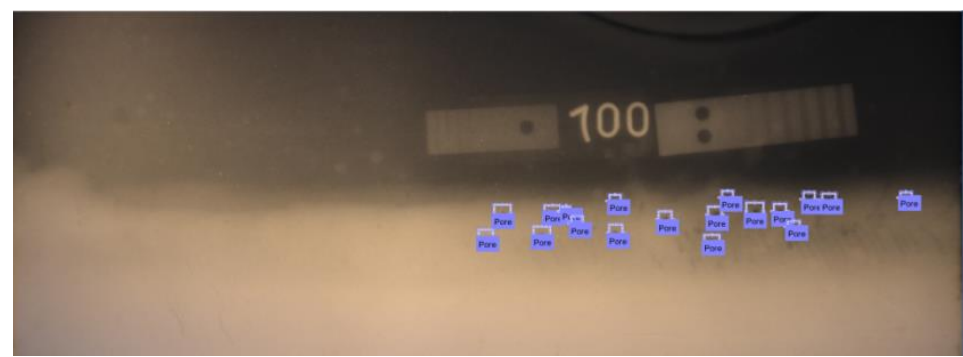

Figure 13. Selection of areas of interest in the form of defects "Pore".

A defect in the form of "Inclusion" is characterized by a darkening area of a fairly large, irregularly shaped. An example of the selection of the area of interest with a defect in the form of inclusion (Inclusion) is presented in Figure 14.

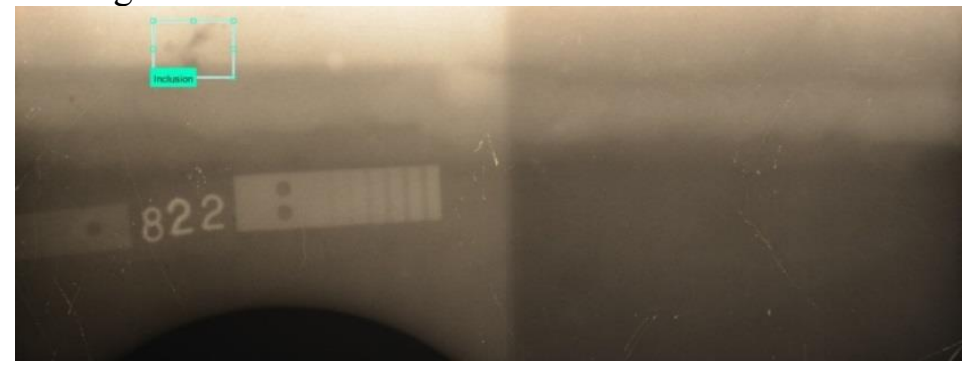

Figure 14. Selection of areas of interest in the form of defects "Inclusion".

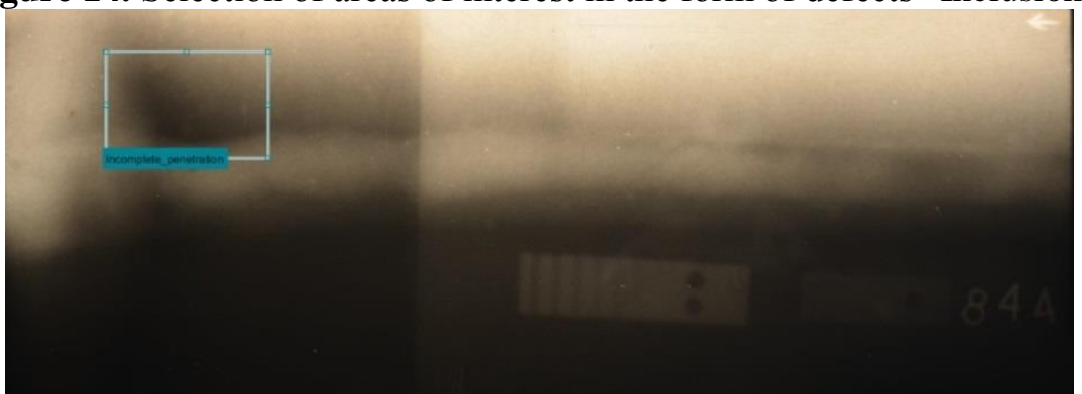

Figure 15. Selection of areas of interest in the form of defects "lack of fusion".

The defect in the form of "lack of fusion" is characterized by a darkening area of sufficiently large size and length, in some cases along the entire length of the weld. An example of the selection of the region of interest with a defect in the form of "lack of fusion" is presented in Figure 15. 
After applying labels of areas of interest to the entire array of images, it is necessary to export the label base using the 'Export Labels' function for further use in training the AlexNet neural network.

\subsection{Pre-processing of images when learning neural network}

Pre-processing includes the following:

- transferring the image to grayscale, smoothing the image using a median filter and noise removing;

- improving image contrast: adaptive histogram equalization.

The obtained image is color for effective detection of defects in the welded joint is necessary to convert the color image into a halftone.

A median filter was used to smooth the image. A $4 \times 4$ matrix is used as a filter mask. Pixels of the original grayscale image corresponding to all elements of the filter mask form an ordered sequence A. Pixel D ( $r, s)$, where $r$ and $c$ are the coordinates of the current position of the center element of the mask, is assigned the value of the median of sequence A. The operation is applied non-recursively for all mask positions $[6,7,21]$.

To improve the image quality, an equalization of the image histogram was used (Figures 16-17).

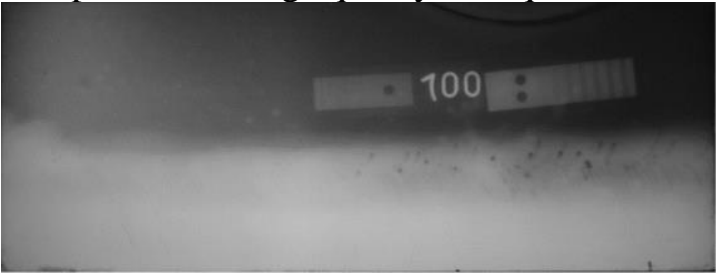

a)

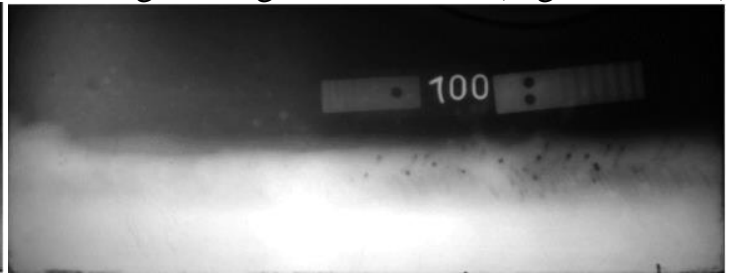

b)

Figure 16. Image of the radiographic image (a) Original; b) improved contrast with gamma correction).

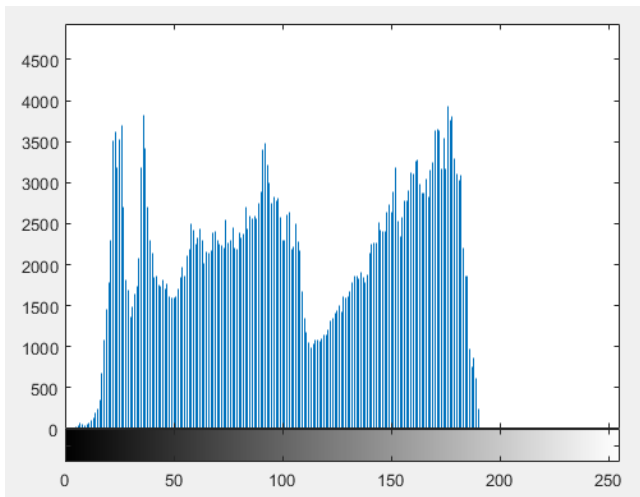

a)

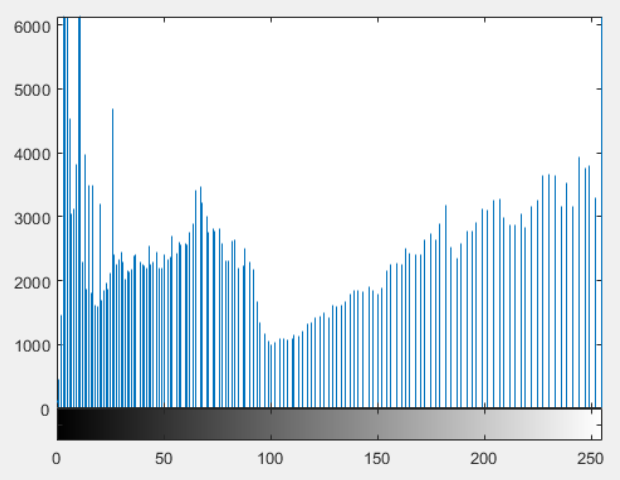

b)

Figure 17. Histogram of the brightness of pixels of the image of the radiographic image (a) of the original; b) improved).

Luminance values $0 \leq \mathrm{i} \leq 255$ are plotted on the abscissa axis, and $0 \leq \mathrm{f}(\mathrm{x}) \leq 1$ along the ordinate axis. If the histogram uses a narrow range of brightness, the equalization method allows you to increase the range of brightness of the image. After equalization of the image, its contrast will increase and the image will contain a greater number of semitones.

\subsection{Defect recognition results}

In this work, the AlexNet convolutional neural network model was implemented and used with the following architecture [7, 19, 20].

In the photographs (Figures 18-22) after the neural network processing, the recognition result is visible, where the area with the recognized defect of the welded joint is highlighted, indicating the degree of confidence in the pattern. 
To determine the accuracy of recognition, it is necessary to find out the number of correct recognition of the neural network for each type of defect (Table 2).

For the convenience of calculating the share of correct recognition in the image, the labels of the region of interest are highlighted (ROI Label). Let's consider several examples of images from a test sample with identified defects to determine the accuracy of the diagnostic system [8-14].

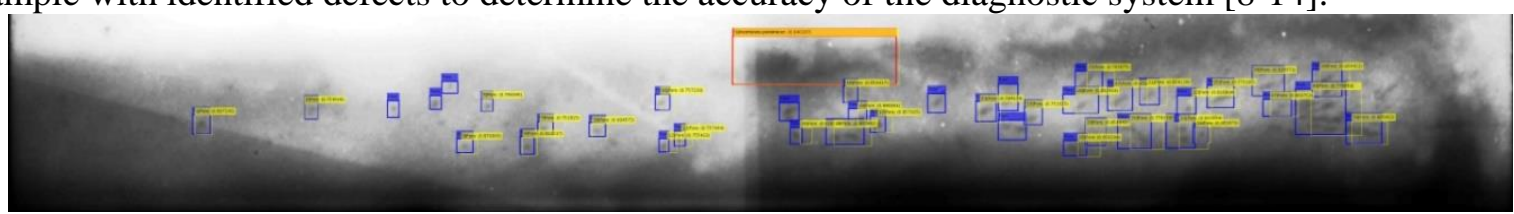

Figure 18. Image No. 1 of a radiographic image with recognized defects like "pore" and "lack of fusion".

For defects found, it is necessary to issue a report and recommendations for repairing the welded joint. An important point is the indication of the possible causes of the defect to enhance the control of the engineering and technical structure of the enterprise over compliance with the welding process and compliance with all technological requirements, which will help avoid the occurrence of defects in welds in the future [15-17].

Conclusion for this snapshot: according to the results, the welded joint is not suitable. It has a large number of defects in the form of "pore" located close enough to each other, just as in the picture there is a defect "lack of fusion".

The cause of the defect in the form of "pore" is the poor quality preparation of the welded edges of the product, the presence of dirt, oil and rust on the surface. Also a possible cause may be poor preparation of the welding material.

The cause of the defect in the form of "lack of fusion" is the incomplete melting of the edges of the base metal due to poor welding material, low quality of the welder, or mismatch of the welding speed and welding current.

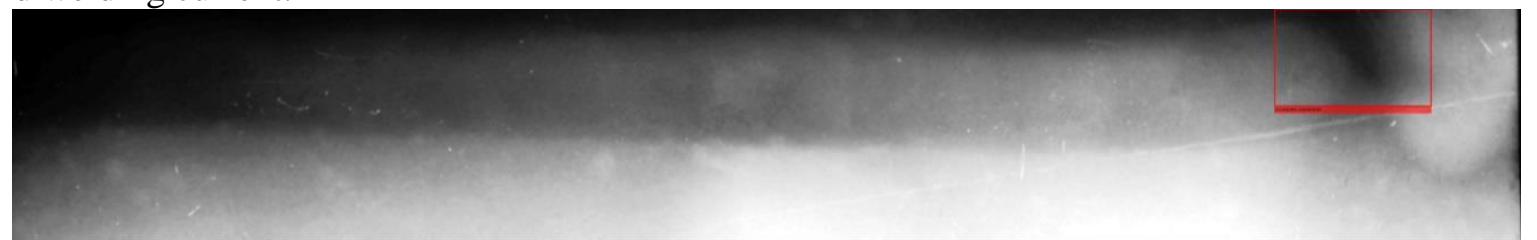

Figure 19. Image No. 2 of the radiographic image with the recognized defect "lack of fusion".

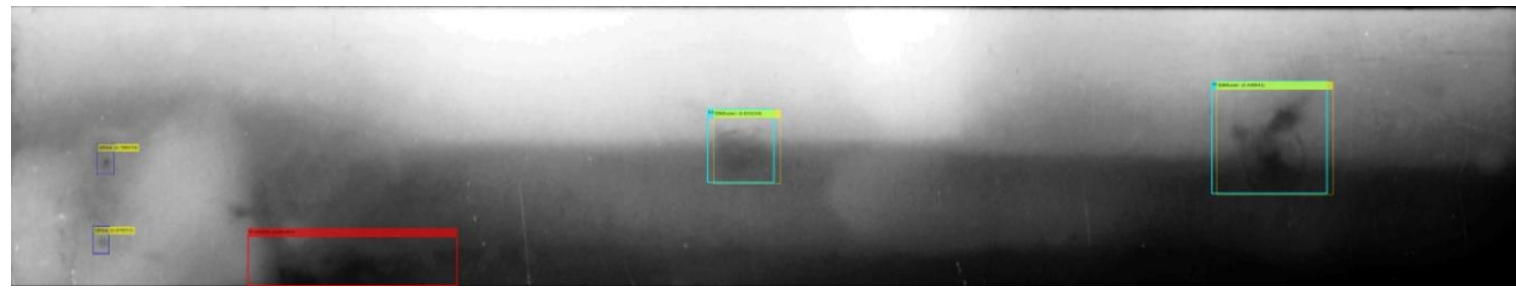

Figure 20. Image No. 3 of a radiographic image with recognized defects of the type "pore", "inclusion" and "lack of fusion".

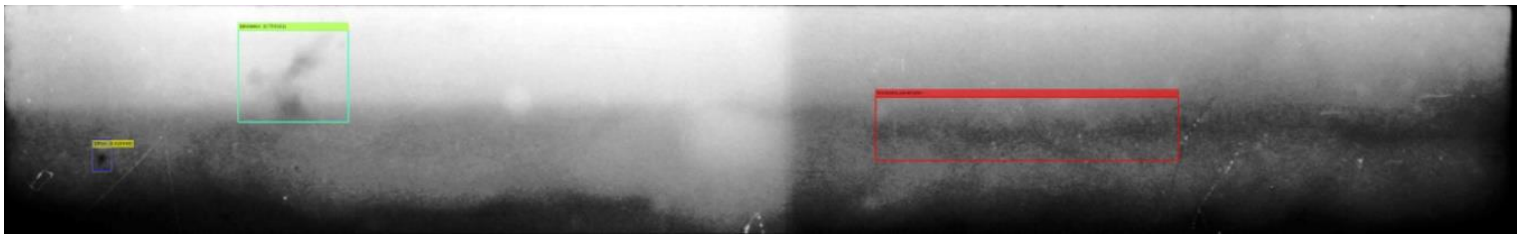

Figure 21. Image No. 4 of a radiographic image with recognized defects of the type "pore", "inclusion" and "lack of fusion". 
In this picture, in addition to defects "pore" and "lack of fusion", there is a defect "inclusion". A possible cause of the occurrence of this defect is poor-quality welding materials, contamination of edges, failure to comply with the welding mode.

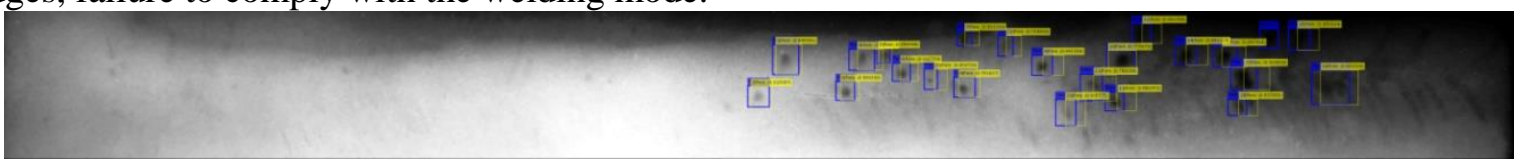

Figure 22. Image No. 5 of a radiographic image with recognized defects of the "Pore" type.

Table 2. Determining the authenticity of recognition for the images.

\begin{tabular}{|c|c|c|c|}
\hline Picture number & Defect name & Amount & Detected \\
\hline \multirow{2}{*}{1} & Pore & 39 & 32 \\
\cline { 2 - 4 } & Lack of fusion & 1 & 1 \\
\hline 2 & Lack of fusion & 1 & 1 \\
\hline \multirow{3}{*}{3} & Pore & 2 & 2 \\
\cline { 2 - 4 } & Inclusion & 2 & 2 \\
\cline { 2 - 4 } & Lack of fusion & 1 & 1 \\
\hline \multirow{3}{*}{4} & Pore & 1 & 1 \\
\cline { 2 - 4 } & Inclusion & 1 & 1 \\
\cline { 2 - 4 } & Lack of fusion & 23 & 22 \\
\hline 5 & Pore & & \\
\hline
\end{tabular}

Let's calculate the total accuracy of the test sample of thirty images of defects in welded joints (Table 3).

Table 3. Determination of the reliability of recognition of defects on the test sample of images [11-

\begin{tabular}{|c|c|c|c|}
\hline \multicolumn{5}{|c|}{ 17] } \\
\hline Defect name & Amount & Detected & Accuracy \\
\hline Pore & 162 & 143 & 0,8827 \\
\hline Inclusion & 15 & 14 & 0,9333 \\
\hline Lack of fusion & 22 & 21 & 0,9545 \\
\hline
\end{tabular}

According to the data obtained (Table 3), it can be concluded that $88.27 \%$ of defects of the first type of pore were correctly classified as pores, $93.33 \%$ of defects of the second type of inclusion (Inclusion) were correctly classified as inclusions, 95.45\% defects of the third type of incomplete (Incomplete_penetration) were correctly classified as incomplete. Overall, 92.35\% of the predictions made by the neural network are correct and $7.65 \%$ are erroneous.

\section{Conclusions}

The paper presents an overview of modern methods of digital image processing and image recognition methods, implemented algorithmic and software, presents the results of modeling a system for detecting defects in welded joints using a neural network.

- The analysis of the methods of obtaining digital images of extended welded joints based on the radiographic method was carried out;

- The typical structures of diagnostic systems and digital image processing algorithms were investigated;

- The structure and principles of functioning of the system for detecting defects in welded joints based on images of radiographic images of welded joints using a neural network have been developed;

- Developed algorithmic and software implementation of the localization of defects in welded joints using a neural network; 
- A simulation of the welded joint defect detection system using a neural network was made.

The proposed methods and software system allow you to automate the process of technical diagnostics of welded joints according to the results of radiographic monitoring. The developed algorithm using the neural network allows recognition of defects in welded joints.

\section{References}

[1] Gorbachev V I, Semenov A P 2007 Radiographic control of welded joints (Moscow: Sputnik) p 486

[2] PNAE G-7-017-89 1990 Unified control methodology of base metals (semi-finished products), welded joints and surfacing of equipment and pipelines. Radiographic control (Moscow)

[3] GOST 30242-97 1997 Defects of joints during fusion welding. Classification, notation and definitions (Moscow)

[4] GOST 7512-82 1982 Non-destructive control. Welded joints. Radiographic method (Moscow)

[5] PNAE G-7-010-89 2000 Equipment and pipelines of nuclear power plants. Welded joints and cladding. Rules of control (Moscow)

[6] Zapata J 2010 An adaptive network based fuzzy inference system for classification of welding defects NDT\&E International 43 191-199

[7] Kozhenkov E V, Lyasin D N 2016 Research of automated analysis methods of welded joints Xrays (Volzhskiy)

[8] Valavanis I, Kosmopoulos D 2010 Multiclass defect detection and classification in weld radiographic images using geometric and texture features Expert Systems with Applications 37 7606-7614

[9] Thiruganam M 2010 Automatic Defect Detection and Counting In Radiographic Weldment Images Int. J. of Comp. Applications 2(10) 1-5

[10] Vilar R, Zapata J and Ruiz R 2009 An automatic system of classification of weld defects in radiographic images NDT\&E International 42 467-476

[11] Wang X, Wong B S and Tan C S 2010 Recognition of Welding Defects in Radiographic Images by Using Support Vector Machine Classifier Research Journal of Applied Sciences, Engineering and Technology 2(3) 295-301

[12] Mahmoudi A, Regragui F 2009 Welding Defect Detection by Segmentation of Radiographic Images IEEE Conf. on World Congress on Comp. Science and Inf. Engineering 111-115

[13] Ghazvini M 2009 Defect Detection of Tiles Using 2D-Wavelet Transform and Statistical Features World Academy of Science, Engineering and Technology 49 901-904

[14] Xiaomeng W 2009 Detection of Weld Line Defect for Oil-gas Pipeline Based on X-rays Image Processing Proc. Int. Symp. on Web Information Systems and Applications 273-275

[15] Bishop C M 2006 Pattern Recognition and Machine Learning (NY.: Springer) p 738

[16] Gonzalez R, Woods R 2008 Digital Image Processing 3rd edition (Prentice Hall) p 976

[17] Phung S L, Bouzerdoum A 2009 MATLAB Library for Convolutional Neural Networks. Technical report (Wollongong) $\mathrm{p} 20$

[18] Gaydel A V, Pervushkin S S 2013 The study of textural signs for the diagnosis of diseases of the bone tissue on X-ray images Computer Optics 37(1) 113-119

[19] Soldatova O P, Garshin A A 2010 Application of convolutional neural network for handwriting recognition Computer Optics 34(2) 252-259

[20] Izotov P Yu, Kazanskiy N L, Golovashkin D L and Sukhanov S V 2011 CUDA-Enable Implementation of a Neural Network Algorithm for Handwritten Digit Recognition Optical Memory and Neural Networks (Information Optics) 20(2) 98-106 DOI: 10.3103/ S1060992X11020032

[21] Kalinovskii I A, Spitsyn V G 2016 Review and testing of frontal face detectors Computer Optics 40(1) 99-111

\section{Acknowledgements}

This work was supported by the Russian Foundation for Basic Research, research № 17-08-01569. 